\title{
Prediction of outcome by prenatal Doppler analysis in a patient with aortic stenosis
}

\author{
P-S Jouk, P Rambaud
}

\begin{abstract}
Stenosis of the aortic valve (pressure drop $50 \mathrm{~mm} \mathrm{Hg}$ ) was diagnosed prenatally by Doppler echocardiography in a 33 week old fetus. Measurement of time-velocity integrals through the tricuspid and mitral valves indicated a significantly higher flow in the right heart. The pressure drop across the aortic valve in the 3 hour old infant was $80 \mathrm{~mm} \mathrm{Hg}$. The findings in this patient suggest that the usually accepted theory that prenatally the ventricles function in parallel should take into account the chronology of filling and ejection. In this patient the ability of a ventricle to generate a prenatal transvalvar pressure gradient was evidence that the size, compliance, and contractility of the ventricle were sufficient to maintain good function.
\end{abstract}

Aortic stenosis is infrequently diagnosed in utero. Most reports are of patients with critical aortic stenosis and poor ventricular dimensions or function or both. ${ }^{1-3}$ In such patients it was difficult prenatally to distinguish between aortic stenosis and primary left ventricular myocardial disease or the hypoplastic left heart syndrome and to decide on the best approach to perinatal management. ${ }^{4}$

Grenoble, Grenoble,

France

P-S Jouk

P Ramboud

Correspondence to

Dr P-S Jouk, Service de

Medicine Neonatale et

Reanimation Infantile,

Centre Hospitalier Regiona

et Universitaire de Grenoble,

38043 Grenoble Cedex

France.

\section{Methods}

Fetal echocardiographic studies were performed with a Hewlett-Packard 77020 ultrasound scanner and a $5 \mathrm{MHz}$ medium focus probe. Doppler ultrasonic intensities

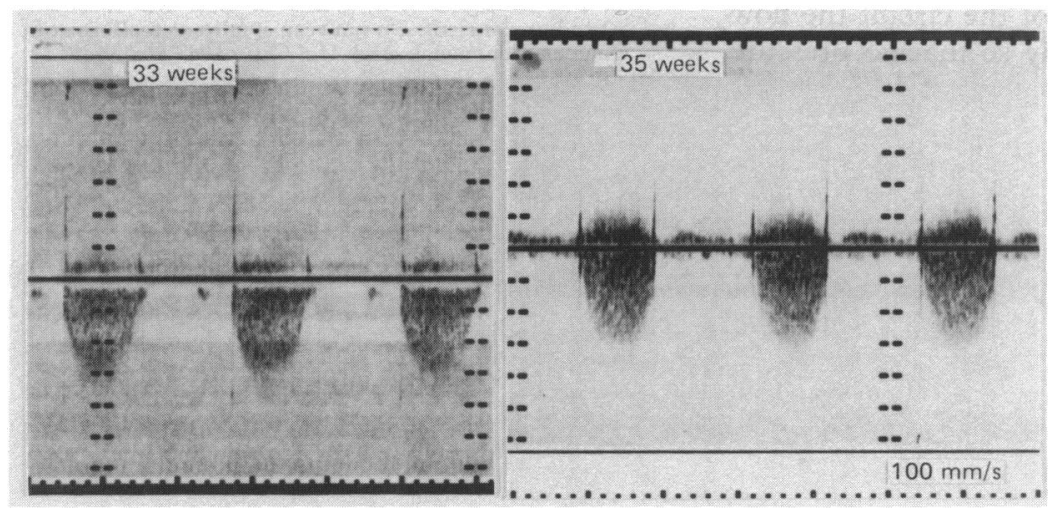

Figure 1 Prenatal diagnosis of aortic valve stenosis in a fetus at 33 and 35 weeks' gestation. Continuous wave Doppler examination shows a peak systolic velocity of $3.5 \mathrm{~m} / \mathrm{s}$ corresponding to a gradient of $50 \mathrm{~mm} \mathrm{Hg}$. were limited to below the $100 \mathrm{~mW} / \mathrm{cm}^{2}$ spatial peak time average. After visualisation of the fetal heart by cross sectional imaging and identification of the best incident angle the continuous wave Doppler probe was used to obtain maximum velocity traces across the aortic valve.

\section{Case report}

A woman was referred to our laboratory at 32 weeks' gestation because of a unexplained late fetal death at 36 weeks during a previous pregnancy. The size of the fetus was appropriate for the gestational age and the fetus was in sinus rhythm with a heart rate of 130 beats/minute. There was no evidence of cardiac failure. The ventricular septum was thicker than normal for the gestational age $(0.45 \mathrm{~cm})$ (normal range $\left.0 \cdot 2-0 \cdot 38^{5}\right)$. Left ventricular function and dimensions were within the normal ranges. The maximal diameters of the tricuspid and mitral orifices were estimated at $12 \mathrm{~mm}$ and $9 \mathrm{~mm}$ respectively. Cross sectional echocardiography showed an unusual pulsatile dilatation in the ascending aorta on the anterior wall suggesting a poststenosis jet lesion. The aortic valve seemed thickened and was dome shaped in systole. Because of the position of the fetus it was impossible to obtain a good Doppler examination of the aortic valve. The mother was therefore re-examined a week later.

At 33 weeks' gestation the fetus was in good position and the maximal transstenotic velocity was $3.5 \mathrm{~m} / \mathrm{s}$ (fig 1 ) (corresponding to a mimimum gradient of about $50 \mathrm{~mm} \mathrm{Hg}$ ). No tricuspid or mitral insufficiency was detectable by colour flow Doppler. The fetus was then examined every 2 weeks to monitor progress.

At 35 weeks' gestation the transvalvar gradient was the same. However, timevelocity integrals through the tricuspid and mitral valves were $8.36 \mathrm{~cm}$ and $4.92 \mathrm{~cm}$ respectively (fig 2). This large difference between the time-velocity integrals when there was only a moderate difference in tricuspid and mitral size indicated a significantly higher flow in the right heart and was predictive of a postnatal increase in gradient. At 38 weeks' gestation and after a normal labour, a male infant $(2730 \mathrm{~g})$ with a normal Apgar score was delivered. Examination 3 hours after delivery showed no signs of cardiac failure. A 3/6 ejection systolic murmur was heard; it was maximal at the upper right sternal edge. Doppler echocardiography showed that the min- 


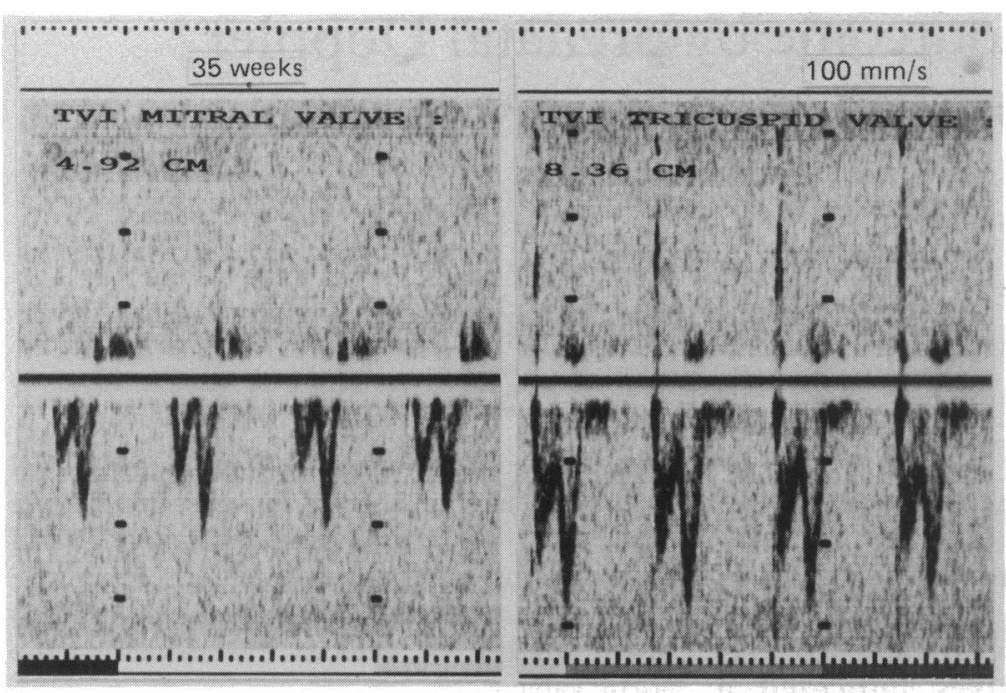

Figure 2 Evaluation of the flow in each ventricle in a 35 week old fetus. The time velocity integral across the mitral valve is $4.92 \mathrm{~cm}$ and that across the tricuspid valve is $8.36 \mathrm{~cm}$ drop is generated. Because the flow is pulsatile rather than continuous, the systolic and diastolic phases should be considered separately. During the diastolic phase the preload is the same for the right and left ventricles if the flow across the foramen ovale is unrestricted, as is usually the case. ${ }^{67}$ Differential ventricular diastolic filling mainly depends on the dimensions and compliance of each ventricle. This diastolic filling becomes a major determinant, along with myocardial contractility, of the systolic ejection volume. In the fetus during the systolic phase the afterload is essentially the same for both ventricles. Therefore, the gradient would be determined by the systolic ejection volume and the stenotic surface area.

This model, which takes into account the chronology of filling and ejection, is more realistic and explains the possibility of a considerable transvalvar pressure gradient in a fetus with an intact ventricular septum. In addition, to generate a pressure drop across the semilunar valve the dimensions, compliance, and contractility of the ventricle must be adequate. In our patient the transvalvar pressure gradient of $50 \mathrm{~mm} \mathrm{Hg}$ was paradoxically a favourable prognostic factor; this accorded with the evidence of good left ventricular function detected during echocardiographic examination of the fetus-that is normal ventricular cavity dimensions and ejection fraction. But measurement of the transvalvar gradient alone is not sufficient to predict the condition in the neonate. This requires evaluation of the flow across the stenotic valve and of the differential flow in each ventricle. ing diastole. A transventricular valvotomy was performed with a $5 \mathrm{~mm}$ Hegar candle. The postoperative gradient was $50 \mathrm{~mm} \mathrm{Hg}$ without valve insufficiency. At the age of one he was symptom free and growing normally.

\section{Discussion}

To our knowledge this is the first reported measurement of a significant transvalvar pressure drop across a stenosed semilunar valve in a fetus. It also shows that such a drop is possible. This suggests that the accepted theory that ventricles function in parallel prenatally may be too simplistic. From this model it follows that when a stenosis is present in one element of the circuit the flow decreases proportionately so that no pressure
1 De Geeter B, Marnange JP, Willard D, et al. Rétrecissement aortique valvulaire. Diagnostic prenatal. Presse Med 1985 14:1505-7.

2 Huhta JC, Carpenter RJ, Moise KJ, et al. Prenatal diagnosis Circulation 1987;75:573-6.

3 Allan LD, Sharland G, Tynan MJ. The natural history of the hypoplastic left heart syndrome. Int J Cardiol 1989; 25:395-8

4 Robertson MA, Byrne PJ, Penkoske PA. Perinatal management of critical aortic valve stenosis diagnosed by fetal echocardiography. Br Heart J 1989;61:356-7. NTP Press, 1986:48-55.

6 Jouk PS, Rossignol AM, Bost M. Cinétique normale et pathologique de la valve du foramen ovale. Etude échocardiographique foetale et doppler pulsé [Abstract]. Clin Invest Med 1987;10:37.

7 Jouk PS, Rossignol AM, Denis B, Bost M. L'ostium secundum restrictif. Un nouveau syndrome malformatif.
Diagnostic anténatal. Arch Mal Coeur 1987;80:538-42. and postnatal management of critical aortic stenosis.

5 Allan LD. Manual of fetal echocardiography. Lancaster: 